\title{
New octane booster molecules for modern gasoline composition
}

\author{
J.H. Badiaa , E. Ramírez ${ }^{a}$, R. Bringuéa ${ }^{2}$ F. Cunilla*, J. Delgado ${ }^{b}$
}

a Chemical Engineering and Analytical Chemistry Department, University of Barcelona, Martí i Franquès 1-11, 08028, Barcelona, Spain

${ }^{\mathrm{b}}$ Repsol Technology Lab., Avenida Agustin de Betancourt, s/n, 28935, Móstoles (Madrid), Spain

*Corresponding author's e-mail: fcunill@ub.edu

\section{Supporting Information}

Table S1. Toxicology associated to considered high-octane gasoline components Globally Harmonized System of Classification and Labelling of Chemicals (GHS) in compliance with Regulation (EC) No 1272/2008.

\begin{tabular}{|c|c|c|c|c|c|c|c|c|c|c|c|c|c|c|c|c|c|c|}
\hline \multirow{3}{*}{$\begin{array}{l}\text { Family } \\
\text { Compound } \\
\end{array}$} & \multirow[b]{3}{*}{ CAS } & \multicolumn{2}{|c|}{ Physical hazards } & \multicolumn{13}{|c|}{ Health hazards } & \multicolumn{2}{|c|}{$\begin{array}{l}\text { Environmental } \\
\text { hazards }\end{array}$} \\
\hline & & \multirow{2}{*}{$\begin{array}{c}\text { Flammable } \\
\text { gases }\end{array}$} & \multirow{2}{*}{$\begin{array}{c}\text { Flammable } \\
\text { liquids }\end{array}$} & \multicolumn{3}{|c|}{ Acute toxicity } & \multirow[t]{2}{*}{$\begin{array}{c}\text { Skin } \\
\text { corrosion }\end{array}$} & \multicolumn{2}{|c|}{ Irritation } & \multirow{2}{*}{$\begin{array}{c}\text { Serious } \\
\text { eye } \\
\text { damage }\end{array}$} & \multirow{2}{*}{$\begin{array}{l}\text { Repro- } \\
\text { ductive } \\
\text { toxicity }\end{array}$} & \multirow{2}{*}{$\begin{array}{c}\text { Germ } \\
\text { cell } \\
\text { muta- } \\
\text { genicity }\end{array}$} & \multirow[t]{2}{*}{$\begin{array}{l}\text { Carcino- } \\
\text { genicity }\end{array}$} & \multicolumn{2}{|c|}{$\begin{array}{l}\text { Specific target } \\
\text { organ toxicity }\end{array}$} & \multirow[t]{2}{*}{$\begin{array}{c}\text { Aspiration } \\
\text { hazard }\end{array}$} & \multirow{2}{*}{$\begin{array}{l}\text { Acute } \\
\text { aquatic } \\
\text { toxicity }\end{array}$} & \multirow{2}{*}{$\begin{array}{l}\text { Chronic } \\
\text { aquatic } \\
\text { toxicity }\end{array}$} \\
\hline & & & & Oral & Inhalation & Dermal & & Skin & Eye & & & & & $\begin{array}{c}\text { single } \\
\text { exposure }\end{array}$ & $\begin{array}{l}\text { repeated } \\
\text { exposure }\end{array}$ & & & \\
\hline \multicolumn{19}{|l|}{ Isoparaffins } \\
\hline \multirow{4}{*}{$\begin{array}{r}\text { iso-butane } \\
\text { iso-pentane } \\
2,3 \text {-di-methyl } \\
\text { butane } \\
2,2,3-\text { tri-methyl } \\
\text { butane } \\
\end{array}$} & $75-28-5$ & 1 & & & & & & & & & & & & \multirow{4}{*}{\multicolumn{2}{|c|}{$\begin{array}{l}3 \\
3 \\
3\end{array}$}} & & & \\
\hline & $78-78-4$ & & 1 & & & & & & & & & & & & & 1 & & 2 \\
\hline & $79-29-8$ & & 2 & & & & & 2 & & & & & & & & 1 & & 2 \\
\hline & $464-06-2$ & & 2 & & & & & 2 & & & & & & & & 1 & 1 & 1 \\
\hline \multicolumn{19}{|l|}{ Olefins } \\
\hline \multirow{3}{*}{$\begin{array}{r}\text { 2-Methyl-1- } \\
\text { butene } \\
2-\text { Methyl-2- } \\
\text { pentene } \\
\text { 2,3-Dimethyl-1- } \\
\text { butene }\end{array}$} & $563-46-2$ & & 1 & & & & & & & & & & & & & 1 & & \\
\hline & $625-27-4$ & & 2 & & & & & & & & & & & & & 1 & & \\
\hline & $563-78-0$ & & 2 & & & & & & & & & & & & & 1 & & \\
\hline
\end{tabular}




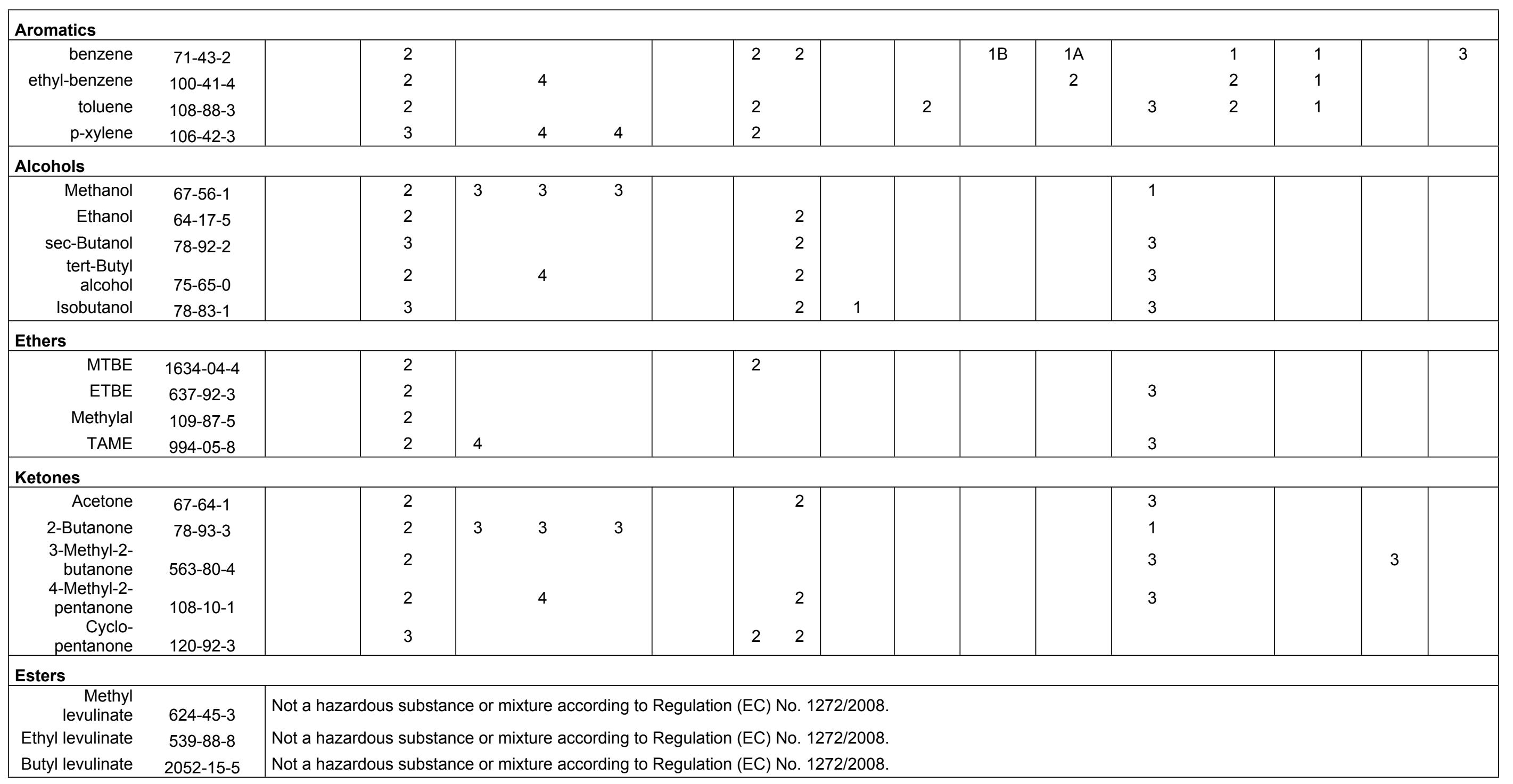




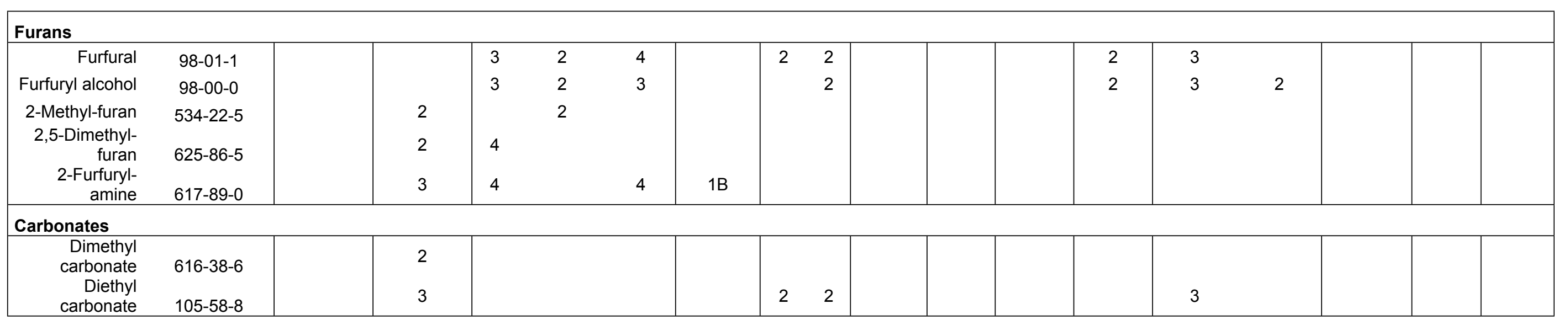


Table S2. Toxicology associated to considered ash-less octane enhancer additives Globally Harmonized System of Classification and Labelling of Chemicals (GHS) in compliance with Regulation (EC) No 1272/2008.

\begin{tabular}{|c|c|c|c|c|c|c|c|c|c|c|c|c|c|c|c|c|c|c|}
\hline \multirow[b]{3}{*}{ Compound } & \multirow[b]{3}{*}{ CAS } & $\begin{array}{l}\text { Physical } \\
\text { hazards }\end{array}$ & \multicolumn{14}{|c|}{ Health hazards } & \multicolumn{2}{|c|}{$\begin{array}{l}\text { Environmental } \\
\text { hazards }\end{array}$} \\
\hline & & \multirow{2}{*}{$\begin{array}{c}\text { Flammable } \\
\text { liquids }\end{array}$} & \multicolumn{3}{|c|}{ Acute toxicity } & \multirow[t]{2}{*}{$\begin{array}{c}\text { Skin } \\
\text { corrosion }\end{array}$} & \multicolumn{2}{|c|}{ Irritation } & \multirow[t]{2}{*}{$\begin{array}{c}\text { Serious } \\
\text { eye } \\
\text { damage }\end{array}$} & \multicolumn{2}{|c|}{ sensitization } & \multirow[t]{2}{*}{$\begin{array}{l}\text { Repro- } \\
\text { ductive } \\
\text { toxicity }\end{array}$} & \multirow{2}{*}{$\begin{array}{c}\text { Germ } \\
\text { cell } \\
\text { muta- } \\
\text { genicity }\end{array}$} & \multirow[t]{2}{*}{$\begin{array}{l}\text { Carcino- } \\
\text { genicity }\end{array}$} & \multicolumn{2}{|c|}{$\begin{array}{l}\text { Specific target } \\
\text { organ toxicity }\end{array}$} & \multirow[t]{2}{*}{$\begin{array}{l}\text { Acute } \\
\text { aquatic } \\
\text { toxicity }\end{array}$} & \multirow[t]{2}{*}{$\begin{array}{l}\text { Chronic } \\
\text { aquatic } \\
\text { toxicity }\end{array}$} \\
\hline & & & Oral & Inhalation & Dermal & & Skin & Eye & & Skin & Respiratory & & & & $\begin{array}{c}\text { single } \\
\text { exposure }\end{array}$ & $\begin{array}{l}\text { repeated } \\
\text { exposure }\end{array}$ & & \\
\hline \multicolumn{19}{|l|}{ Anilines } \\
\hline Aniline & $62-53-3$ & & 3 & 3 & 3 & & & & 1 & 1 & & & 2 & 2 & & 1 & 1 & 1 \\
\hline $\mathrm{N}$-methyl-aniline & $100-61-8$ & & 3 & 3 & 3 & & & & & & & & & & & 2 & 1 & 1 \\
\hline $\begin{array}{l}\text { 2,4-Dimethyl- } \\
\text { aniline }\end{array}$ & $95-68-1$ & & 3 & 3 & 3 & & & & & & & & & & & 2 & & 2 \\
\hline Diphenylamine & $122-39-4$ & & 3 & 3 & 3 & & & & & & & & & & & 2 & 1 & 1 \\
\hline o-Toluidine & $95-53-4$ & & 1B & 3 & 3 & & & & 2 & & & & & & & & 1 & \\
\hline $\begin{array}{l}2,4,6-\text { Trimethyl-n- } \\
\text { methylaniline }\end{array}$ & $\begin{array}{c}13021-14- \\
2\end{array}$ & & & & 2 & & & & 2 & & & & & & & & 1 & \\
\hline $\begin{array}{l}\mathrm{N}, \mathrm{n} \text { '-di-sec-butyl-p- } \\
\text { phenylene-diamine }\end{array}$ & $101-96-2$ & & 3 & & 2 & & & & 1 & 1 & & & & & & & 1 & 1 \\
\hline \multicolumn{19}{|l|}{ Hydrazine } \\
\hline Hydrazine & $302-01-2$ & & 3 & 3 & & 1B & & & & 1 & & & & 1B & & & 1 & 1 \\
\hline UDMH & $57-14-7$ & 2 & 3 & 3 & 3 & 1B & & & & & & & & 1B & 3 & & & 2 \\
\hline $\mathrm{MMH}$ & $60-34-4$ & 2 & 2 & 1 & 2 & $1 \mathrm{~B}$ & & & & & & & & 1B & & & 1 & 1 \\
\hline $\mathrm{DPH}$ & $122-66-7$ & & 4 & & & & & & & & & & & $1 \mathrm{~B}$ & & & 1 & 1 \\
\hline \multicolumn{19}{|l|}{ Amines } \\
\hline $\begin{array}{r}\text { 4-sec-butyl-o- } \\
\text { phenylene diamine }\end{array}$ & $95-54-5$ & & 3 & 4 & 4 & & & 2 & & 1 & & & 2 & 2 & & & 1 & 1 \\
\hline Isopropyl amine & $75-31-0$ & 1 & 3 & 3 & 3 & $1 \mathrm{~A}$ & & & & & & & & & 3 & & & \\
\hline Ethylene diamine & $107-15-3$ & 3 & 4 & 4 & 3 & 1B & & & 1 & 1 & 1 & & & & & & & 3 \\
\hline
\end{tabular}


Diethyl amine

\begin{tabular}{l|l|l}
$109-89-7$ & 2 & 4 \\
\hline
\end{tabular}

Pyridines

\begin{tabular}{|c|c|c|c|c|c|c|c|c|c|c|c|c|c|c|c|c|}
\hline 2-methyl-pyridine & $109-06-8$ & 3 & 4 & 4 & 3 & & 2 & & & & & & 3 & & & \\
\hline \multicolumn{17}{|l|}{ Quinolines } \\
\hline $\begin{array}{r}\text { quinoline } \\
1,2,3,4- \\
\text { tetrahydroquinoline } \\
\end{array}$ & $\begin{array}{l}91-22-5 \\
635-46-1 \\
\end{array}$ & & 3 & & 4 & $\begin{array}{l}2 \\
2\end{array}$ & $\begin{array}{l}2 \\
2\end{array}$ & & & & 2 & $\begin{array}{l}1 \mathrm{~B} \\
1 \mathrm{~B}\end{array}$ & 3 & & & 2 \\
\hline \multicolumn{17}{|l|}{ Indoles } \\
\hline Indole & $120-72-9$ & & 4 & & 3 & 2 & & 1 & & & & & 3 & & 1 & \\
\hline \multicolumn{17}{|l|}{ Nitrosamines } \\
\hline $\begin{array}{r}\text { n-nitroso- } \\
\text { diphenylamine } \\
\text { n-nitroso-n- } \\
\text { methylaniline }\end{array}$ & $\begin{array}{l}86-30-6 \\
614-00-6 \\
\end{array}$ & & $\begin{array}{l}4 \\
3\end{array}$ & & & & 2 & 2 & & & & 2 & & & 2 & \\
\hline \multicolumn{17}{|l|}{ lodine compounds } \\
\hline $\begin{array}{r}\text { iodine } \\
\text { methyl iodide } \\
\text { ethyl iodide }\end{array}$ & $\begin{array}{c}7553-56-2 \\
74-88-4 \\
75-03-6\end{array}$ & & $\begin{array}{l}3 \\
4\end{array}$ & $\begin{array}{l}4 \\
3\end{array}$ & $\begin{array}{l}4 \\
4\end{array}$ & $\begin{array}{l}2 \\
2 \\
2\end{array}$ & $\begin{array}{l}2 \\
2 \\
\end{array}$ & & 1 & 1 & & 2 & $\begin{array}{l}3 \\
3 \\
3\end{array}$ & 1 & 1 & \\
\hline
\end{tabular}

Selenium compounds

$\begin{array}{rr}\text { selenium } & 7782-49-2 \\ \text { diphenyl selenide } & 1132-39-4 \\ \text { diphenyl diselenide } & 1666-13-3 \\ \text { selenium } & \\ \text { oxychloride } & 7791-23-3 \\ \text { selenium } & 10026-03-\end{array}$

selenium 10026-03-

\begin{tabular}{|l|ll|l|l|l|l|}
3 & 3 & & & & & \\
-3 & 3 & 3 & & & & \\
-3 & 3 & 3 & & & & \\
\hline 3 & 3 & 3 & & & & \\
\hline
\end{tabular}
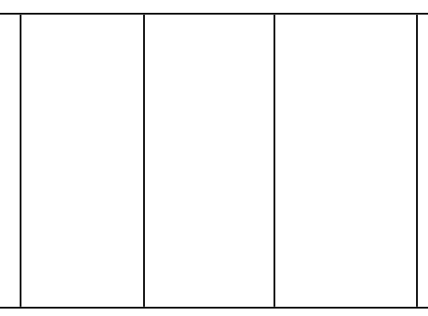

(12)

\begin{tabular}{l|l|l|}
2 & & 4 \\
2 & 1 & 1 \\
2 & 1 & 1 \\
2 & 1 & 1 \\
2 & 1 & 1 \\
\hline
\end{tabular}

\section{Phenols}

$\begin{array}{rr}\text { Phenol } & 108-95-2 \\ \text { p-Cresol } & 106-44-5 \\ 2,4-X y l e n o l & 105-67-9 \\ 4-E t h y l-p h e n o l & 123-07-9\end{array}$

\begin{tabular}{|lll|l|}
3 & 3 & 3 & $1 \mathrm{~B}$ \\
3 & & 3 & $1 \mathrm{~B}$ \\
3 & & 3 & $1 \mathrm{~B}$ \\
& & & $1 \mathrm{C}$
\end{tabular}

$\mid$

$\left|\begin{array}{l|l|}2 & \\ & \end{array}\right|$

2 


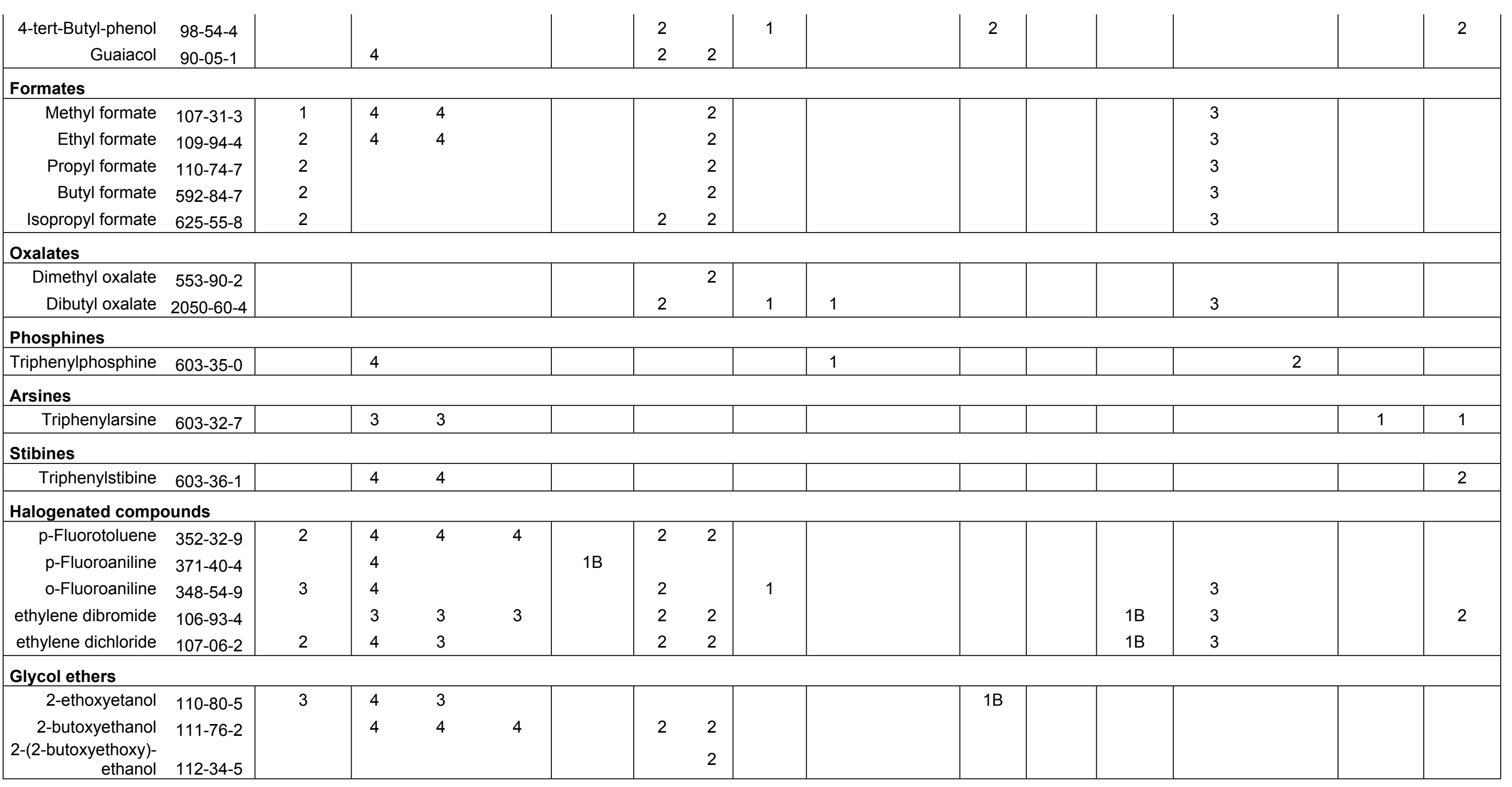

